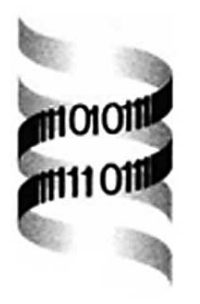

\title{
How to decide whether small samples comply with an equidistribution
}

\author{
Thorsten Pöschel ${ }^{1}$ and Jan A Freund ${ }^{2}$ \\ ${ }^{1}$ Institut für Biochemie, Charité, Monbijoustraße 2, Berlin, D-10117, Germany and \\ ${ }^{2}$ Institut für Physik, Humboldt-Universität zu Berlin, Invalidenstraße 110, Berlin, \\ D-10115, Germany
}

\begin{abstract}
Motivation: The decision whether a measured distribution 'complies with an equidistribution is a central element of many biostatistical methods. High throughput differential 'expression measurements, for instance, necessitate to judge possible over-representation of genes. The reliabil'ity of this judgement, however, is strongly affected when rarely expressed genes are pooled. We propose a method 'that can be applied to frequency ranked distributions and that yields a simple but efficient criterion to assess the 'hypothesis of equiprobable expression levels.

Results: By applying our technique to surrogate data we 'exemplify how the decision criterion can differentiate between a true equidistribution and a triangular distribution. 'The distinction succeeds even for small sample sizes where standard tests of significance (e.g. $\chi^{2}$ ) fail. Our method will have a major impact on several problems of computational biology where rare events baffle a reliable assessment of frequency distributions.
\end{abstract}

Availability: The program package is available upon 'request from the authors.

Contact: thorsten.poeschel@charite.de

\section{INTRODUCTION}

Biostatistical analyses quite generally infer desired infor'mation from experimentally observed frequency distributions. Microarrays, for instance, can screen thousands of 'genes simultaneously, thus allowing for high-throughput measurements Gershon, 2002. The expression level of 'genes is quantified via hybridisation signal intensities and, after an appropriate normalisation procedure, yields a vector of numbers which can be understood as an empirically obtained frequency distribution. The important issues of coregulation (Kielbasa et al., 2001) and differential expression (Tsodikov et al., 2001) are based on further analysis of these distributions. In this context, pooling a family of rarely expressed genes can be combined with the task to decide whether the observed expression pattern complies with an equidistribution (Tandle et al., 2001). As is intuitively clear, the small sample size makes this decision a hard problem - nobody would expect to reproduce the statistics of a fair die (with equal probability $1 / 6$ for each side) with only four trials. The problem of small sample statistics pervades literature, e.g. (Storey et al., 1998; Helland et al., 1998; Josefsson et al., 1998). Similar problems might occur in the statistical analyses of codon distributions (Som et al., 2001) or other biopolymers (Ramsden and Vohradský, 1998). As a last field of potential application let us mention the computational comparison of two draft sequences of the human genome Aach et al., 2001).

We propose a method to decide the above posed question of an equidistribution based on frequency ranked statistics. The technique yields a criterion that can detect frequency distributions generated from a true equidistribution and reject others. It is important to note that the criterion we have devised is rather efficient for small sample sizes (expression levels) where standard measures of significance like the $\chi^{2}$-test or the Kolmogorov-Smirnov test fail. We expect that biostatistical analyses of small sample data, as met e.g. for rarely expressed genes, can profit from our criterion.

\section{SYSTEM AND METHODS}

Assume, in an experimental sampling probe we find $N^{*}$ different species occurring with relative frequencies $f_{1}$, $f_{2} \ldots f_{N^{*}}$. The term species is not meant in its strict biological sense here but more general as a class of individuals. As an example we mention the subsequences of a certain length, e.g. $l=6$, of the nuclein acids $A, G$, $C$, and $T$. A species is the word $G A T A G G$ which may be found in a gene at various positions, e.g., at positions 45,122 , and 431. For this example we say that the species GAT AGG occurs with 3 representatives.

Quite often uniform probabilities are motivated by theoretical considerations or as the simplest assumption. We present a practical criterion based on finite sample size statistics that allows to decide, i.e. to accept or reject, the hypothesis of an underlying uniform probability distribution $p_{1}=p_{2}=\ldots=p_{N}=1 / N$ with $N \geq N^{*}$.

Given a probability distribution $\left\{p_{i}, i=1, \ldots, N\right\}$ with $\sum_{i=1}^{N} p_{i}=1$ where the $p_{i}$ represent the likelihood to find a representative of species $i$ from a set of $N$ 
possible species. Assume, in an experiment we find $M_{1}$ copies of species $1, M_{2}$ copies of species $2 \ldots M_{N^{*}}$ copies of species $N^{*}$, with $\sum_{i=1}^{N^{*}} M_{i}=M$ and none of the remaining species $N^{*}+1, \ldots, N$. The fact that not all of the $N$ species might be observed means $N^{*} \leq N$. Stated differently, the number $N$ is not directly accessible by the experiment but has to be estimated using probabilistic arguments. However, beyond all methods of optimally estimating the unknown $N$ - which then completely specifies the assumed equidistribution - the question arises how probable the hypothesis of an equidistribution is at all.

Clearly, the law of large numbers asserts the stochastic convergence of relative frequencies towards the related probabilities, i.e.

$$
p_{i} \equiv \lim _{M \rightarrow \infty} f_{i}, \quad f_{i} \equiv \frac{M_{i}}{M}
$$

where the limit is approached for almost all (in the mathematical sense) experimental realizations (sampling probes). For cases where the condition $M \gg N$ is not fulfilled, however, the relative frequencies often deviate considerably from the related probabilities, e.g. Schmitt et al. (1993); Herzel et al. (1994); Pöschel et al. (1995). Strictly speaking, for finite $M$ one cannot even be sure to have found each species at least once. Just imagine one or a few species with probabilities being orders of magnitude smaller than the overwhelming rest of nearly identically probable species. One might say that in such a situation all the tiny probability events are dispensable for an efficient description and a uniform distribution is true rather in a practical sense. However, in other situations deviations of an underlying probability distribution from a true equidistribution can be that significant that the hypothesis of a uniform probability distribution should be judged as inappropriate. As an example consider a triangle shaped distribution. Now, how does this substantial discrepancy show up in experimental sampling probes and how can this be distinguished from pure finite sample size effects?

To illustrate typical distortions of the equidistribution due to finite sample size we depict Fig. 1 (left) which shows a frequency distribution obtained by drawing $M=$ $10^{4}$ equidistributed random integers from the interval $[1,2, \ldots 1000]$, i.e., $N=1000$. From the probabilities $p_{1}=p_{2}=\ldots p_{N}=10^{-3}$ we expect to find each of the numbers, on average, $M / N$ times, i.e., $\left\langle f_{1}\right\rangle=$ $\left\langle f_{2}\right\rangle=\ldots=\left\langle f_{N}\right\rangle=10$. Figure 11 shows that there are large fluctuations of the occurrences of the numbers. A more convenient way to represent these data is the rank ordered frequency distribution. To this end we reorder the abscissa in a way to receive a decaying curve of frequencies, i.e., the most frequent species occupies rank 1 , the most frequent but one occupies rank 2 etc. The rank ordered frequencies are depicted in the right part of Fig. 1.
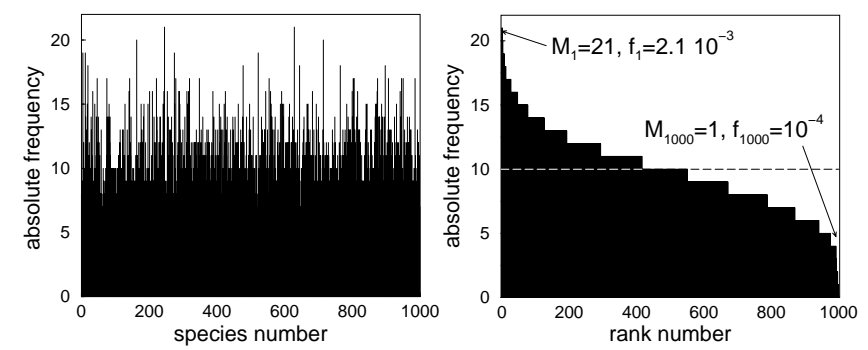

Fig. 1. The measured frequency distribution may deviate from the probability distribution considerably due to finite sample effects. The left figure shows absolute frequencies which have been obtained by drawing $M=10^{4}$ random integers from the interval $1 \ldots N, N=1000$. The right figure presents the same data but in rank order (see text). Whereas the expectation value for each of the numbers is $M / N=10$ (dashed line) large deviations are noticeable.

Since our random numbers are equidistributed by construction the expectation value for each of the numbers (ranks) is $M / N=10$. As seen from Fig. 11 fluctuations around this mean are considerable: if one naively inferred the probabilities (or concentrations) from the relative frequencies, one would end up with a relative error of $110 \%$ for small ranks and $90 \%$ for large ranks. Being faced with a measurement as the one sketched in Fig. 1 it is far from trivial to decide whether the objects (in our case random numbers) are equidistributed. It is the aim of this paper to propose a method which allows to distinguish between uniform and non-uniform probability distributions if the sample size is too small to identify the probabilities with the relative frequencies due to Eq. (11).

\section{Finite size statistics}

For equidistributed events $j \in[1 \ldots N]$ with $p_{j}=p=$ $1 / N$ the probability to find with $M$ trials a number of at least $k_{i}$ different events each occurring exactly $i$ times with $i=0, \ldots, M$ reads (von Mises, 1939)

$$
P\left(k_{i}, i\right)=\frac{M !}{(i !)^{k_{i}}\left(M-k_{i} i\right) !}\left(\frac{1}{N}\right)^{k_{i} i}\left(1-\frac{k_{i}}{N}\right)^{\left(M-k_{i} i\right)} .
$$

Applying the Exclusion-Inclusion-Principle (Johnson and Kotz, 1977) to Eq. (2) one can derive the probability to find with $M$ trials exactly $k_{i}$ different species each occurring exactly $i$ times:

$$
\begin{aligned}
& p\left(k_{i}, i\right)=\sum_{j=k_{i}}^{\lfloor M / i\rfloor}(-1)^{\left(j-k_{i}\right)}\left(\begin{array}{c}
j \\
k_{i}
\end{array}\right) P(j, i) \\
& =\frac{M !}{N^{M}} \sum_{j=k_{i}}^{\lfloor M / i\rfloor}(-1)^{\left(j-k_{i}\right)}\left(\begin{array}{c}
j \\
k_{i}
\end{array}\right) \frac{(N-j)^{(M-j i)}}{(i !)^{j}(M-j i) !}
\end{aligned}
$$


where $\lfloor x\rfloor$ stands for the integer of $x$. The first moment of this probability reads

$$
\left\langle K_{i}\right\rangle=\left(\begin{array}{c}
M \\
i
\end{array}\right) N^{(1-i)}\left(1-\frac{1}{N}\right)^{(M-i)} .
$$

Hence, when randomly drawing $M$ representatives each occurring with the same probability $p_{1}=p_{2}=$ $\ldots p_{N}$, one expects to find $\left\langle K_{0}\right\rangle$ species zero-times, $\left\langle K_{1}\right\rangle$ once, $\ldots\left\langle K_{M}\right\rangle$ species $M$ times. The full derivation of Equations (3) and (A), using rather involved algebra, can be found in Freund and Pöschel (1995); Pöschel and Freund (1997).

For our above example, if $M$ random integers have been drawn from the interval $1, \ldots, N$, Eq. (4) describes how many random numbers, on average, occur exactly zero times, i.e., never $\left(\left\langle K_{0}\right\rangle\right)$, once $\left(\left\langle K_{1}\right\rangle\right)$, twice $\left(\left\langle K_{2}\right\rangle\right)$, .., $M$ times $\left(\left\langle K_{M}\right\rangle\right)$. Hence, using Eq. ( $\boxplus$ ), for an equidistribution it is possible to calculate the expected measured frequencies (Pöschel and Freund, 2002).

In converse direction, it is possible to infer for each $i=$ $1 \ldots M$ a value $N^{(i)}$ from the experimentally observed $k_{i}$ by identifying $k_{i}=\left\langle K_{i}\right\rangle(i=1 \ldots M)$ and making use of Eq. (4). Thus, sampling a true equidistribution, one should find

$$
N^{(1)} \approx N^{(2)} \approx \ldots \approx N^{(L)} \approx N
$$

In theory, the distribution (3) exists for $i=1 \ldots M$ where the event related to $k_{M}$ corresponds to the extreme case that all $M$ representatives belong to the same species. In measurements not all $k_{i}$ can be different from zero. Therefore, the approximative equation (5) is valid for all upper indices $(L)$ for which the corresponding $k_{L} \neq 0$ has been found in the measurement.

The estimated values read for $i=1$ :

$$
N^{(1)}=\left[1-\left(\frac{\left\langle K_{1}\right\rangle}{M}\right)^{\frac{1}{M-1}}\right]^{-1}
$$

and for all other occupation numbers $\left\langle K_{i}\right\rangle$

$$
N^{(i)}=\left[\left(\begin{array}{c}
M \\
i
\end{array}\right) \frac{1}{\left\langle K_{i}\right\rangle}\left(1-\frac{1}{N^{(i)}}\right)^{M-i}\right]^{i-1} .
$$

Equation (7) has to be solved numerically by an iteration procedure. As discussed below in dependence on the variables $M,\left\langle K_{i}\right\rangle$, and $i$ this equation may have zero, one, or two solutions and one has to select the appropriate one.

To clarify the meaning of the approximate identity signs in Eq. (5) we point out that the identification $k_{i}=\left\langle K_{i}\right\rangle$ is an approximation which should be amended by statistical fluctuations, i.e., $k_{i}=\left\langle K_{i}\right\rangle+\left\langle\Delta K_{i}\right\rangle$, with $\left\langle\Delta K_{i}\right\rangle \sim$ $\sqrt{\operatorname{var} K_{i}}$. The variance of $K_{i}$ for an equidistribution can be achieved analytically using the generating function of $p\left(k_{i}, i\right)$ (see Eq. (3)). It reads (Pöschel and Freund, 2002)

$$
\begin{aligned}
& \operatorname{var} K_{i}=\left\langle K_{i}^{2}\right\rangle-\left\langle K_{i}\right\rangle^{2} \\
& \quad=\left\langle K_{i}\right\rangle\left[1+\left(\begin{array}{c}
M-i \\
i
\end{array}\right) \frac{(N-2)^{M-2 i}}{(N-1)^{M-i-1}}\right]-\left\langle K_{i}\right\rangle^{2} .
\end{aligned}
$$

This variance of the $K_{i}$ can be converted into a characteristic error interval around the derived $N_{i}$ simply by applying Eq. (円) not only to $K_{i}$ but also to $K_{i}+\Delta K_{i}$ and $K_{i}-\Delta K_{i}$.

This means, if for each $i$ we plot the set of experimentally determined numbers $N^{(i)}$ together with their expected range of fluctuations $\left( \pm \sqrt{\operatorname{var} N_{i}}\right)$, for an underlying equidistribution we should be able to draw a straight line which passes through all the error intervals. On the contrary, if any horizontal line significantly falls outside at least one of the intervals the hypothesis of an underlying equidistribution should be rejected.

\section{RESULTS}

We want to illustrate the method by means of an equidistribution

$$
p_{i}=1 / 100, \quad i=1, \ldots, 100
$$

and a triangular distribution

$$
p_{i}=\frac{2}{100}\left(1-\frac{i}{101}\right), \quad i=1, \ldots, 100 .
$$

As discussed above, when drawing random events according to these probability distributions, the resulting rank-ordered frequency distributions will significantly depend on the sample size $M$. Figure 2 shows the rankordered frequency distributions for different values of the sample size $M$. Whereas the top row figures $\left(M=10^{4}\right)$ clearly reflect the equidistribution (left) and the triangular distribution (right), respectively, for smaller sample sizes (lower rows) one notices, as expected, significant deviations between probabilities and frequencies. The central issue is now whether from these small sample rank-ordered frequency distributions (lower rows) one can nevertheless distinguish between the uniform and the triangular probability distribution.

For all plots drawn in Fig. 2 2 we calculated the estimated total number of events $N^{(i)}$ from the observed occupation numbers $k_{i}$ for diverse cluster sizes $i$. The result is shown in Fig. 2. As expected, only for the equidistributed species (left plots) we find that the approximate constancy of $N^{(i)}$ as expressed by Eq. (5) holds true. Surprisingly, even for quite small sample size $M=100$, i.e., very poor statistics, we can clearly distinguish between the frequency distributions originating from the equidistribution Eq. (9) and 
those which originate from the triangular distribution Eq.

(10).
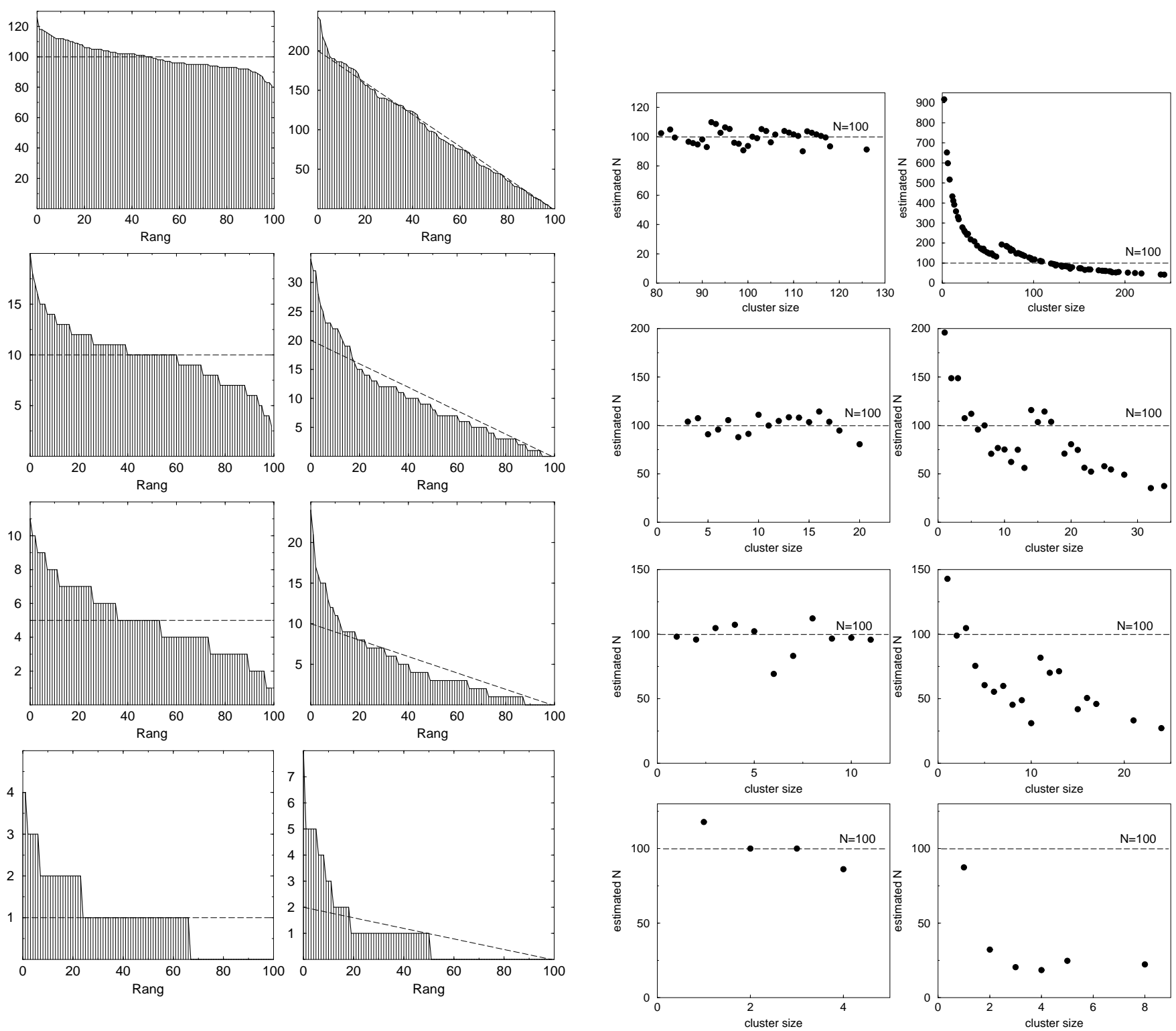

Fig. 2. Frequencies generated from the equidistributed probabilities, Eq. (19) (left plots) and from the triangular probability distribution Eq. 10) for different sample sizes. From top to bottom: $M=10^{4}, M=10^{3}, M=500, M=100$. The dashed lines show the underlying probability distributions, Eq. 99) (left figures) and Eq. (10), respectively.

Figure 7 shows the mean

$$
N_{\mathrm{est}} \equiv \frac{\sum_{i=1}^{M} N^{(i)}}{\#\left(k_{i}\right)}
$$

of the estimated total number of species and the standard

Fig. 3. Estimated total number of species due to Eqs. (6) and ( the data shown in Fig. . For equidistributed species (left figures) we find the condition Eq. (5) fulfilled, whereas for triangularly distributed species different occupation numbers $k_{i}$ yield significantly different estimates $N^{(i)}$. From top to bottom: $M=10^{4}, M=10^{3}, M=500, M=100$. The dashed lines display the correct value $N=100$. 
deviation

$$
s \equiv \sqrt{\frac{\sum_{i=1}^{M}\left(N^{(i)}\right)^{2}}{\#\left(k_{i}\right)}-\left(\frac{\sum_{i=1}^{M} N^{(i)}}{\#\left(k_{i}\right)}\right)^{2}}
$$

for the equidistribution over the sample size $M$. The summation in Eqs. (11) and (12) runs over all indices $i \in$ $[1, M]$ which correspond to occupation numbers $k_{i} \neq 0$. The symbol \# $\left(k_{i}\right)$ stands for the number of these nonempty occupations. The larger the sample size the smaller the standard deviation. Even for rather small sample sizes $M \approx 150$ the estimated total number of species agrees well with the true value $N=100$ as shown in the zoomed region (lower plot in Fig. \#). The dashed lines show the true total number of species, $N=100$.
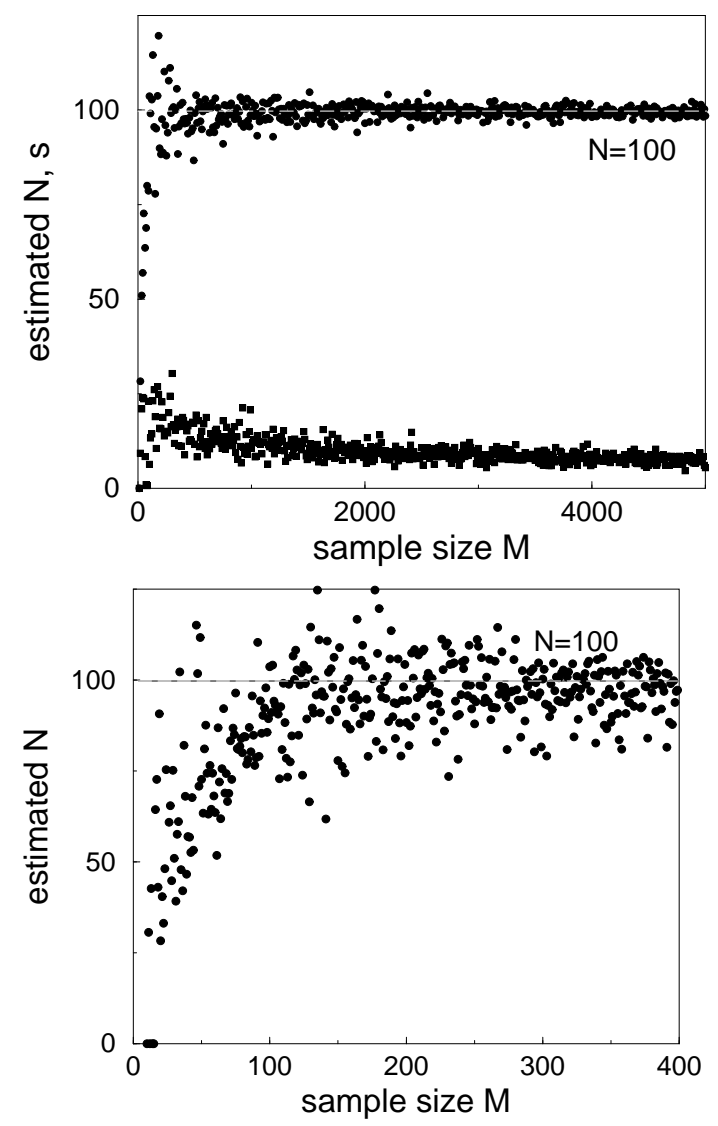

Fig. 4. The mean estimated total number of species (circles) and the standard deviation $s$ (boxes) due to Eqs. (11) and (12) for species distributed according to the equidistribution Eq. (9). The dashed lines show the true total number of species $N=100$.

Figure 5 depicts the corresponding data for species which were generated from the triangular probability distribution Eq. (10). For larger sample sizes $M$ we obtain a growing mean of the estimated total number of species. With increasing sample size the standard deviation increases too.

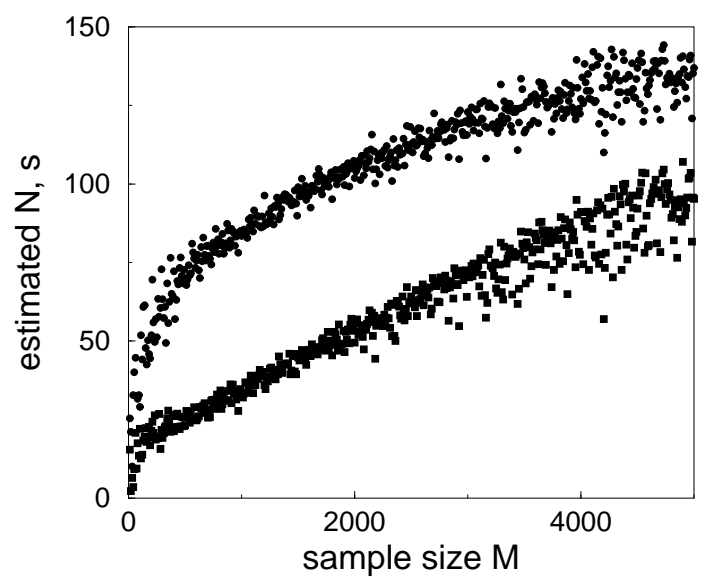

Fig. 5. The mean estimated total number of species (circles) and the standard deviation $s$ (boxes) due to Eqs. 111) and (12) for species distributed according to the triangular distribution Eq. (10).

\section{SOLUTIONS OF EQ. (7)}

From Eq. (4) it follows that for a given sample size $M$ the curve $\left\langle K_{i}\right\rangle$, now understood as a function of the total number of species $N$, has a maximum. As an example, in Fig. 6 we plotted $\left\langle K_{20}\right\rangle$ vs. $N$ (for $M=1000$ ). The curve has a maximal value $\left\langle K_{20}\right\rangle^{\max } \approx 4.6$ at $N \approx 52.6$, meaning that there is no uniform probability distribution which is in agreement with any larger experimentally determined values of $k_{20}$.

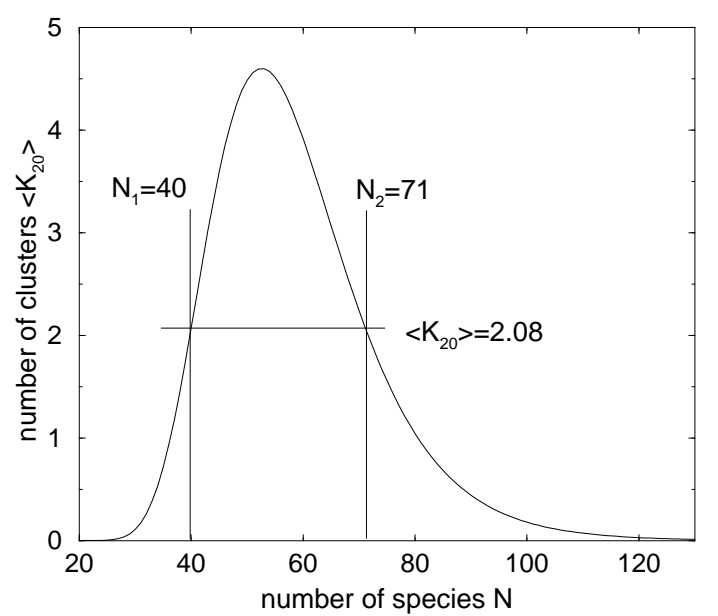

Fig. 6. $\left\langle K_{20}\right\rangle$ over the total number of species $N$ for $M=1000$. The curve has a maximum $\left\langle K_{20}\right\rangle^{\max } \approx 4.6$ at $N \approx 52.6$.

The extremum of $\left\langle K_{i}\right\rangle$ for $i \geq 2$ can be found from Eq. 
(4):

$$
\left\langle K_{i}\right\rangle^{\max }=\left(\begin{array}{c}
M \\
i
\end{array}\right)(1-M)^{1-M}(i-M)^{M-i}(1-i)^{i-1}
$$

which occurs for

$$
N^{\max }=\frac{M-1}{i-1} .
$$

Hence, Eq. (7) has no solution for any $k_{i}>\left\langle K_{i}\right\rangle^{\max }$. Figure 6 shows also that for any $k_{i}<\left\langle K_{i}\right\rangle^{\max }$ there are two solutions of Eq. (7): for $M=1000$ the value $k_{20}=2$ has the solutions near $N_{1}=40$ and $N_{2}=71$. The reason for this behaviour becomes clear when drawing $\left\langle K_{i}\right\rangle$ over $i$ for $N_{1}=40$ and $N_{2}=71$ (Fig. 77). The curves intersect at $i=20$ in agreement with the two solutions of Eq. (7). In the same way each value of $k_{i}<\left\langle K_{i}\right\rangle^{\max }$

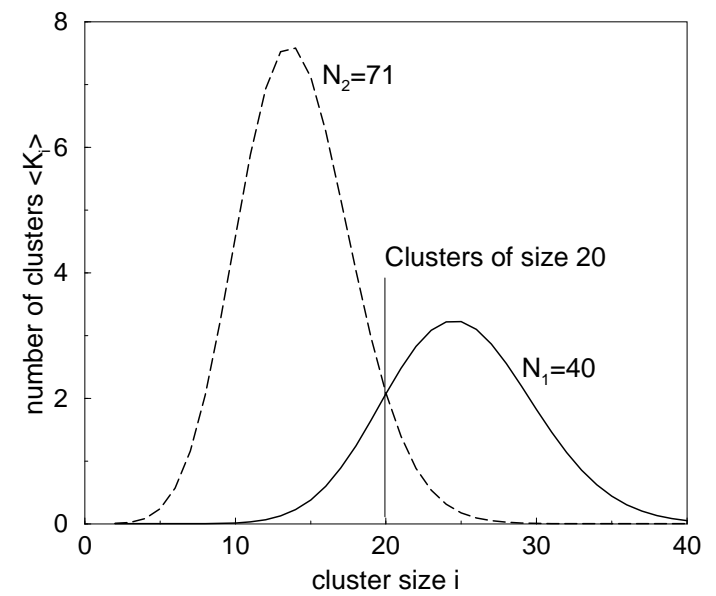

Fig. 7. $\left\langle K_{i}\right\rangle$ over $i$ due to Eq. (4) for $M=1000$ and $N_{1}=40$ (full line) and $N=71$ (dashed line), respectively. The lines intersect at $i=20$.

corresponds to two such solutions, $N_{1}^{(i)} \leq N_{2}^{(i)}$. Hence, for a construction of the plots in Fig. 3 one always has to choose the appropriate branch. If we had only one measurement $k_{i}$ for a particular $i$ there were no means to figure out whether the corresponding $N$ is $N_{1}$ or $N_{2}$. In our case, however, there is a series of $k_{i}$ for a certain range of $i$ and all of them originate from the same measurement.

Obviously, the size $k_{i}$ of the largest $i$ which is found in the data corresponds to the right branch of the curve $\left\langle K_{i}\right\rangle(i)$ as drawn in Fig. 7, i.e., to $N_{2}^{(i)}$. If it would correspond to the left branch, larger $i$ should still be populated. Contrary, the smallest $i$ correspond to $N_{1}^{(i)}$. From the single humped-shape of the curve $\left\langle K_{i}\right\rangle(i)$ we conclude that there is only one transition from $N_{1}$ to $N_{2}$, i.e., the solution is

$$
N^{(i)}=\left\{\begin{array}{lll}
N_{2}^{(i)} & \text { for } & i \leq i^{*} \\
N_{1}^{(i)} & \text { for } & i>i^{*}
\end{array}\right.
$$

where $i^{*}$ has to be determined. For an equidistribution we expect that $N^{(i)}$ for all $i$ approximate the true $N$ (see Eq. (5)). Therefore, we choose $i^{*}$ from $\left[i_{\min }, i_{\max }\right]$ such that it minimises

$$
(\Delta N)^{\left(i^{*}\right)} \equiv \sqrt{\overline{\left(N^{\left(i^{*}\right)}\right)^{2}}-\left(\overline{N^{\left(i^{*}\right)}}\right)^{2}} \rightarrow \min ,
$$

with

$$
\begin{aligned}
\overline{N^{\left(i^{*}\right)}} & \equiv \frac{\sum_{i=i_{\min }}^{i^{*}} N_{2}^{(i)}+\sum_{i=i^{*}+1}^{i_{\max }} N_{1}^{(i)}}{i_{\max }-i_{\min }+1} \\
\overline{\left(N^{\left(i^{*}\right)}\right)^{2}} & \equiv \frac{\sum_{i=i_{\min }}^{i^{*}}\left(N_{2}^{(i)}\right)^{2}+\sum_{i=i^{*}+1}^{i_{\max }}\left(N_{1}^{(i)}\right)^{2}}{i_{\max }-i_{\min }+1}
\end{aligned}
$$

and with $i_{\min }$ and $i_{\max }$ being the sizes of the smallest and largest clusters which are found in the data set.

The estimated number $N$ which is drawn in Figs. 3. 4, and 5 is, hence, given by Eq. (15) with the condition (16). Figure (8) drafts the described procedure.
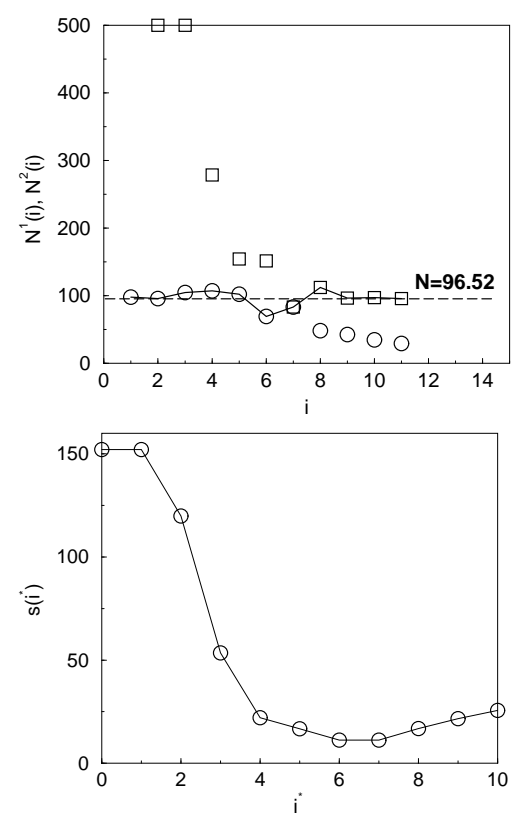

Fig. 8. Top: Both solutions of Eq. ( (7), $N^{1}(i)$ and $N^{2}(i)$ for the equidistribution $M=500$ and $N=100$, corresponding to Fig. (3) (3rd row, left column). The choice $i^{*}=7$ minimises $s$ (see bottom figure) and yields $\bar{N} \approx 96.52$.

\section{DISCUSSION}

Given a sampling probe of size $M$ originating from an unknown probability distribution, it may be important to decide whether the data set is compatible with an equidistribution with known or unknown total number of species 
$N$. Due to finite sample size effects, experimentally accessible frequency distributions will always experience deviations from the underlying probability distribution - be it uniform or non-uniform. Rank-ordering the frequency distribution, being a first step towards a systematic survey, still does not help for rather small sample sizes. The challenge is to find a criterion, solely based on the frequency distribution (data set), which allows to accept or reject the hypothesis of a uniform probability distribution and, in case of acceptance, to render the number $N$ within statistical errors.

We developed a method based on an analytic expression for the average number of events $\left\langle K_{i}\right\rangle$ occurring $i$ times (the varying lengths of the plateaus in the rank-ordered frequency distributions) which involves the sample size $M$ and the a priori (un)known number $N$. By inversion of this relation it is possible to compute for each $i$ an estimate $N^{(i)}$ for the hypothetical $N$, completely specifying the assumed equidistribution. For true uniform probability distributions these estimates should vary slightly, i.e. within expected statistical errors, when varying $i$. Substantial variations of the estimates, i.e. beyond expected statistical errors, or trends are a clear signature of a nonuniform distribution.

We exemplified this method by applying it to a uniform and a triangular distribution. The separation between both by virtue of our criterion is possible down to small sample sizes for which visual inspection of rankordered frequency distributions or standard tests such as $\chi^{2}$ and Kolmogorov-Smirnov do not allow for a clear-cut distinction.

ACKNOWLEDGEMENTS We thank Werner Ebeling and Cornelius Frömmel for discussion.

\section{REFERENCES}

Aach, J., M. L. Bulyk, G. M. Church, J. Comander, A. Derti, and J. Shendure (2001). Computational comparison of two draft sequences of the human genome. Nature 409, 856-859.

Freund, J. A. and T. Pöschel (1995). A statistical approach to vehicular traffic. Physica A 219, 95-114.

Gershon, D. (2002). Microarray technology: An array of opportunities. Nature 416, 885-891.

Helland, Å., A. Langerød, H. Johnsen, A. O. Anne Olsen, E. Skovlund, and A.-L. Børresen-Dale (1998). p53 polymorphism and risk of cervical cancer. Nature 396, 530-531.

Herzel, H., A. O. Schmitt, and W. Ebeling (1994). Finite sample effects in sequence analysis. Chaos, Solitons \& Fractals 4, 97 113.

Johnson, J. N. and S. Kotz (1977). Urn Models and Their Application. New York: Wiley.

Josefsson, A. M., P. K. E. Magnusson, N. Ylitalo, P. QuarforthTubbin, J. Pontén, H. O. Adami, and U. B. Gyllensten (1998). p53 polymorphism and risk of cervical cancer. Nature 396, 531.

Kielbasa, S. M., J. O. Korbel, D. Beule, J. Schuchhardt, and H. Herzel (2001). Combining frequency and positional infor- mation to predict transcription factor binding sites. Bioinformatics 17, 1019-1026.

Pöschel, T., W. Ebeling, and H. Rosé (1995). Guessing probability distributions from small samples. J. Stat. Phys. 80, 1443-1452.

Pöschel, T. and J. A. Freund (1997). Cluster statistics and traffic on a lattice. In L. Schimansky-Geier and T. Pöschel (Eds.), Stochastic Dynamics, Volume 484 of Lecture Notes in Physics, pp. 220-231. Berlin: Springer.

Pöschel, T. and J. A. Freund (2002). Finite-sample frequency distributions originating from an equiprobability distribution. preprint.

Ramsden, J. J. and J. Vohradský (1998). Zipf-like behavior in prokaryotic protein expression. Phys. Rev. E 58, 7777-7780.

Schmitt, A. O., H. Herzel, and W. Ebeling (1993). A new method to calculate higher order entropies from finite samples. Europhys. Lett. 28, 303-309.

Som, A., S. Chattopadhyay, J. Chakrabarti, and D. Bandyopadhyay (2001). Codon distributions in DNA. Phys. Rev. E 63, 051908105190818.

Storey, A., M. Thomas, A. Kalita, C. Harwood, D. Gardiol, F. Mantovani, J. Breuer, I. M. Leigh, G. Matlashewski, and L. Banks (1998). Role of a p53 polymorphism in the development of human papilloma-virus-associated cancer. Nature 393, 229-234.

Tandle, A. T., V. Sanghvi, and D. Saranath (2001). Determination of 553 genotypes in oral cancer patients from india. $B r . J$. Cancer 84, 739-742.

Tsodikov, A., A. Szabo, and D. Jones (2001). Adjustments and measurements of diffferential expression for microarray data. Bioinformatics 18, 251-260.

von Mises, R. (1939). Über Aufteilungs- und Besetzungswahrscheinlichkeiten. Rev. Fac. Sci. Univ. Istanbul 4, 145. 